\title{
Inhibition of ERK1/2 improves lipid balance in rat macrophages via $\mathrm{ABCA1/G1}$ and $\mathrm{CD36}$
}

\author{
XIE-HUA XUE $^{1 *}$, FENG-FEI SHI $^{2 *}$, TONG CHEN $^{2}$, WEI WEI $^{1}$, XIAO-MAO ZHOU $^{2}$ and LI-DIAN CHEN ${ }^{2}$ \\ ${ }^{1}$ Department of Neurology, Rehabilitation Hospital Affiliated to Fujian University of Traditional Chinese Medicine; \\ ${ }^{2}$ Institute of Rehabilitation Medicine, Rehabilitation Technology Collaborative Innovation Center, \\ College of Rehabilitation Medicine, Fujian University of Traditional Chinese Medicine, \\ Fuzhou, Fujian 350003, P.R. China
}

Received February 8, 2015; Accepted December 7, 2015

DOI: $10.3892 / \mathrm{mmr} .2015 .4697$

\begin{abstract}
ATP-binding cassette transporters A1 (ABCA1) and G1 (ABCG1), and macrophage scavenger receptor, cluster of differentiation (CD)36, function as key mediators of cholesterol efflux and influx from macrophages. In addition, they are associated with foam cell formation and the development of atherosclerosis (AS). The aim of the present study was to investigate the effects of extracellular signal-regulated kinases $1 / 2$ (ERK1/2) inhibition on lipid balance in oxidized-low-density lipoprotein (Ox-LDL)-stimulated rat macrophages, and to examine the role of ERK1/2 inhibitors in AS. Rat peritoneal macrophages were treated with Ox-LDL alone or in combination with an ERK1/2 inhibitor, U0126, and untreated cells served as controls. Ox-LDL-induced lipid accumulation was detected by DiI fluorescence and oil red $\mathrm{O}$ staining. In addition, the mRNA and protein expression levels of ABCA1, ABCG1 and CD36 were determined using polymerase chain reaction and western blotting, respectively. Treatment with Ox-LDL significantly increased lipid accumulation and upregulated
\end{abstract}

Correspondence to: Professor Li-Dian Chen, Institute of Rehabilitation Medicine, Rehabilitation Technology Collaborative Innovation Center, College of Rehabilitation Medicine, Fujian University of Traditional Chinese Medicine, 1 Qiuyang Road, Minhoushangjie, Fuzhou, Fujian 350003, P.R. China

E-mail: cld@fjtcm.edu.cn

*Contributed equally

Abbreviations: AS, atherosclerosis; RPMs, rat peritoneal macrophages; Ox-LDL, oxidized-low-density liprotein; DiI-ox-LDL, DiI-labeled oxidized-low-density lipoprotein; ABCA1, ATP-binding cassette transporter A-1; ABCG1, ATP-binding cassette transporter G-1; MAPK, mitogen-activated protein kinase; ERK1/2, extracellular signal-regulated kinases 1/2; p-ERK, phosphorylated ERK; PBS, phosphate-buffered saline

Key words: extracellular signal-regulated kinases 1/2, macrophage, lipid metabolism, ATP-binding cassette transporter A-1, CD36, ATP-binding cassette transporter G-1 the mRNA and protein expression levels of ABCA1, ABCG1 and CD36 in macrophages. The addition of U0126 resulted in a marked reduction of lipid deposition, upregulation of ABCA1/G1 expression and suppression of CD36 expression in Ox-LDL-stimulated macrophages. The results of the present study indicated a novel association between ERK1/2 signaling and lipid metabolism, thus suggesting that inhibition of ERK1/2 may be considered a promising therapeutic strategy against AS.

\section{Introduction}

Atherosclerosis (AS) is a progressive disease characterized by accumulation of lipids and fibrous elements in the arterial tunica intima, which results in atherosclerotic plaque formation and arterial narrowing (1). During the progression of AS, macrophages are critical (2). The development of AS is strongly associated with engulfment of oxidized-low-density lipoprotein (Ox-LDL) by macrophages. It has previously been reported that macrophages engulf Ox-LDL via scavenger receptors, resulting in the deposition of a large quantity of lipids, which further promote the progression of AS (1). Recently, mitogen-activated protein kinase (MAPK) signaling cascades have been investigated in macrophages in vitro, in order to evaluate their importance (3).

Extracellular signal-regulated kinases 1/2 (ERK1/2) are members of the MAPK family, which are involved in mediating various processes, including cell cycle progression, cell migration, survival, differentiation and proliferation (4). However, the association between ERK1/2 signaling and lipid metabolism remains to be elucidated.

ERK1/2 inhibitors have been reported to synergize with liver $\mathrm{X}$ receptor (LXR) ligand to induce ATP-binding cassette transporter (ABC)A1 expression and regulate cholesterol efflux (5). In addition, ABCA1 and ABCG1 have been demonstrated to stimulate the efflux of lipids via the reverse cholesterol transport (RCT) pathway (6). As a specific macrophage scavenger receptor, cluster of differentiation (CD)36 recognizes and internalizes Ox-LDL particles, resulting in cellular cholesterol uptake, lipid accumulation in intracellular space and the production of foam cells (7). It has therefore been hypothesized that ABCA1/G1 and CD36 
are crucial to the maintenance of intracellular cholesterol stability or the efficacy of the RCT process (8). However, the regulation of $\mathrm{ABCA} 1 / \mathrm{G} 1$ and $\mathrm{CD} 36$ expression by ERK1/2 in macrophages remains to be elucidated. The aim of the present study was to investigate the association between ERK1/2 and lipid metabolism, and evaluate the effect of ERK1/2 on the expression levels of ABCA1/G1 and CD36 in macrophages.

\section{Materials and methods}

Animals. A total of 40 male Sprague Dawley rats (weight, 200-220 g; age, 8-9 weeks) were purchased from the Laboratory Animal Center of Fujian Medical University (Fuzhou, China). The rats were housed under a $12 \mathrm{~h}$ light/dark cycle at $37^{\circ} \mathrm{C}$ in an atmosphere containing $5 \% \mathrm{CO}_{2}$, and were given ad libitum access to food and water. All animal experiments in the present study were performed in accordance with the recommendations of the Guidelines for the Care and Use of Laboratory Animals of the Ministry of Science of People's Republic of China. The present study was approved by the Animal Care and Use Committee and Institutional Review Board of Fujian University of Traditional Chinese Medicine (Fuzhou, China) (permission no. IRB201100117) and the Ethics Review Committee of Fujian University of Traditional Chinese Medicine (permission no. FJZYYDXERC2011010).

Isolation and treatment of rat peritoneal macrophages (RPMs). The rats were anesthetized with $10 \%$ chloral hydrate (Peking Union-Biology Co., Ltd., Beijing, China) and were sacrificed by cervical dislocation, and soaked in $75 \%$ ethanol for $5 \mathrm{~min}$. RPMs were harvested by lavaging the peritoneal cavity with $10 \mathrm{ml}$ Dulbecco's modified Eagle's medium/Ham's F12 (DMEM-F12; Hyclone; GE Healthcare Life Sciences, Logan, UT, USA). Cells were centrifuged at $4^{\circ} \mathrm{C}, 1,000 \mathrm{xg}$ for $15 \mathrm{~min}$, and cultured in DMEM supplemented with $1 \%$ glutamine and penicillin/streptomycin (Gibco; Thermo Fisher Scientific, Inc., Waltham, MA, USA), and 10\% fetal bovine serum (FBS; Hyclone; GE Healthcare Life Sciences) at $37^{\circ} \mathrm{C}$ in an incubator containing $5 \% \mathrm{CO}_{2}$. RPMs in the exponential growth phase were seeded onto 6 -well plates at a density of $5 \times 10^{6}$ cells/well for the indicated time. RPMs were randomly assigned to three groups. Cells in the control group were incubated in DMEM-F12 supplemented with $10 \%$ FBS for 12, 24 and $48 \mathrm{~h}$; cells in the Ox-LDL group were treated with $50 \mathrm{mg} / \mathrm{l}$ Ox-LDL (Peking Union-Biology Co., Ltd.) or $10 \mu \mathrm{g} / \mathrm{ml}$ DiI-Ox-LDL (Peking Union-Biology Co., Ltd.) for 12,24 and $48 \mathrm{~h}$, respectively (8-12); whereas cells in the Ox-LDL + U0126 group were incubated in DMEM-F12 containing 10\% FBS supplemented with $10 \mu \mathrm{M}$ U0126 (Sigma-Aldrich, St. Louis, MO, USA) and Ox-LDL or DiI-Ox-LDL for 12, 24 or $48 \mathrm{~h}$.

Morphological observation. RPMs were incubated with DMEM-F12 containing 10\% FBS in 6-well plates. Once the cells had adhered to the culture plates, RPMs were pretreated with Ox-LDL alone, or in combination with U0126, for 12, 24 and $48 \mathrm{~h}$, whereas the untreated cells served as controls. Cellular lipid accumulation was detected using oil red $\mathrm{O}$ staining and DiI fluorescence. Cells were washed three times with phosphate-buffered saline (PBS), fixed in 5\% formalin solution for $15 \mathrm{~min}$ at room temperature, washed once with PBS and stained with oil red O (Wuhan Boster Biological Technology, Ltd., Wuhan, China) for $10 \mathrm{~min}$, followed by hematoxylin (Wuhan Boster Biological Technology, Ltd.) staining for $5 \mathrm{~min}$. Cells were then observed under a Leica DM IL compact inverted stereo microscope (Leica Microsystems $\mathrm{GmbH}$, Wetzlar, Germany), and images were captured at magnification $x 400$. In addition, RPMs in monolayer cultures were exposed to DiI-Ox-LDL alone, or in combination with U0126, for 12, 24 and $48 \mathrm{~h}$. The medium was removed and cells were washed with PBS, mounted on cover slips and analyzed using fluorescent microscopy.

Cytotoxicity assay. The viability of RPMs post-treatment with Ox-LDL (10, 30, 50, 70 and $90 \mathrm{mg} / \mathrm{l})$ was assessed using an MTS assay (Promega Corporation, Madison, WI, USA). The RPMs were grown in 96-well plates and were incubated with Ox-LDL (10,30, 50, 70 or $90 \mathrm{mg} / \mathrm{l})$ for 12,24 or $48 \mathrm{~h}$. The MTS assay was used to measure the viability of the cells. Cells were incubated at $37^{\circ} \mathrm{C}$ with MTS $(1.90 \mathrm{mg} / \mathrm{ml})$ for $4 \mathrm{~h}$, and absorbance was measured using a microplate reader (Multiskan GO; Thermo Fisher Scientific Inc.) at a wavelength of $490 \mathrm{~nm}$. Three wells were set for each concentration of Ox-LDL. All experiments were repeated twice.

Reverse transcription-polymerase chain reaction (RT-PCR) assay. Total RNA was isolated from the RPMs using TRIzol ${ }^{\circledR}$ (Invitrogen; Thermo Fisher Scientific, Inc.), and total RNA (500 ng) was reverse transcribed in a total volume of $20 \mu \mathrm{l}$ containing oligo (dT) primers at $42^{\circ} \mathrm{C}$ for $30 \mathrm{~min}$ using TransScript ${ }^{\circledR}$ One-Step gDNA Removal and cDNA Synthesis SuperMix (Beijing Transgen Biotech Co., Ltd., Beijing, China). Primers used for PCR (Thermo Fisher Scientific, Inc.) are presented in Table I. PCR was performed on an Applied Biosystems ${ }^{\circledR} 2720$ Thermal Cycler (Applied Biosystems; Thermo Fisher Scientific, Inc.) in a $20 \mu \mathrm{l}$ reaction system containing cDNA (500 ng/ $\mu \mathrm{l})$, each specific primer, and $2 \mathrm{X}$ EasyTaq $^{\circledR}$ PCR SuperMix (Beijing Transgen Biotech Co., Ltd.) under the following conditions: Pre-denaturation at $94^{\circ} \mathrm{C}$ for 5 min; 35 cycles at $94^{\circ} \mathrm{C}$ for $30 \mathrm{sec}, 58^{\circ} \mathrm{C}$ for $30 \mathrm{sec}$ and $72^{\circ} \mathrm{C}$ for $1 \mathrm{~min}$; followed by final extension at $72^{\circ} \mathrm{C}$ for $7 \mathrm{~min}$. The PCR products were separated by $1.5 \%$ agarose gel electrophoresis, and the relative mRNA expression levels were determined as the ratio of the grayscale value of the target gene to GAPDH (Image-Lab version 5.0; Bio-Rad Laboratories, Inc., Hercules, CA, USA). The experiment was repeated three times.

Western blot analysis. RPMs were digested with pancreatin (Gibco; Thermo Fisher Scientific, Inc.), pippetted evenly, and centrifuged. The supernatant was transferred to a $1.5 \mathrm{ml}$ centrifuge tube, lysed on ice in radioimmunoprecipitation assay lysis buffer (Beyotime Institute of Biotechnology, Shanghai, China) for $30 \mathrm{~min}$, and fractionated three times with ultrasound. The mixture was then centrifuged at $13,800 \mathrm{x} \mathrm{g}$ for $15 \mathrm{~min}$, and the supernatant was stored at $-80^{\circ} \mathrm{C}$ for subsequent experiments. Protein isolated from the RPMs was quantified using the Bicinchoninic Acid assay (Wuhan Boster Biological Technology, Ltd). Approximately $30 \mu \mathrm{g}$ protein was separated by $10 \%$ sodium dodecyl sulfate-polyacrylamide gel electrophoresis at $110 \mathrm{~V}$ for $2.5 \mathrm{~h}$ at room temperature, and 
Table I. Sequences of primers used for reverse transcription-polymerase chain reaction.

\begin{tabular}{ll}
\hline Gene & \multicolumn{1}{c}{ Primer sequence, $5^{\prime} \rightarrow 3^{\prime}$} \\
\hline CD36 & F: ACTCCAGAACCCAGACAACCAC \\
& R: ACCAAGTAAGACCATCTCAACCAG \\
ABCA1 & F: CCTAAGCATTATCAAGGAGGGAAG \\
& R: AGAGATGACAAGGAGGACGGAAG \\
ABCG1 & F: GTCCTGGGCATCTTCTTCATCTC \\
& R: CCAACTCAGCCAACACTCCTCTC \\
GAPDH & F: ACGGCAAGTTCAACGGCACAG \\
& R:GAAGACGCCAGTAGACTCCACGAC
\end{tabular}

CD36, cluster of differentiation 36; ABCA1, ATP-binding cassette transporter A-1; ABCG1, ATP-binding cassette transporter G-1; GAPDH, glyceraldehyde 3-phosphate dehydrogenase; F, forward; R, reverse.

was then transferred to polyvinylidene difluoride membranes (EMD Millipore, Billerica, MA, USA) for a further $1.5 \mathrm{~h}$ at $110 \mathrm{~V}$ and $4^{\circ} \mathrm{C}$. The membrane was blocked with $5 \%$ non-fat milk in Tris-buffered saline for $1 \mathrm{~h}$ at room temperature and probed with mouse monoclonal anti-ABCA1 $(1: 1,000$; cat. no. ab18180), rabbit monoclonal anti-ABCG1 $(1: 1,000$; cat. no. ab52617), rabbit polyclonal anti-CD36 (1:500; cat. no. ab78054) (Abcam, Cambridge, UK), rabbit polyclonal phosphorylated (p)-p44/42 MAPK (ERK1/2) (1:1,000; cat. no. 9101), rabbit polyclonal p44/42 MAPK (ERK1/2) (1:1,000; cat. no. 9102$)$ and anti- $\beta$-actin $(1: 1,000$; cat. no. 4967) (Cell Signaling Technology, Inc., Danvers, MA, USA) antibodies at $4^{\circ} \mathrm{C}$ overnight. The membrane was then washed with PBS, and incubated with horseradish peroxidase-conjugated goat anti-rabbit (1:5,000; cat. no. A0208) and anti-mouse (1:5,000; cat. no. A0216) secondary antibodies (Beyotime Institute of Biotechnology) for a further $2 \mathrm{~h}$ at room temperature. Enhanced chemiluminescence reagents (Beyotime Institute of Biotechnology) and chemiluminescent substrates were used to visualize immunoreactive bands. The grayscale values of the protein bands were estimated using the image processing software Image-Lab version 5.0 (Bio-Rad Laboratories, Inc.). The ratio of the grayscale value of the target protein band to $\beta$-actin protein band was defined as the protein expression level. All experiments were repeated three times.

Statistical analysis. All data are presented as the mean \pm standard deviation. Differences between means were determined using one-way analysis of variance followed by Dunnett's test, or Student's t-test. Statistical analyses were conducted using SPSS version 18.0 (SPSS, Inc., Chicago, IL, USA). $\mathrm{P}<0.05$ was considered to indicate a statistically significant difference.

\section{Results}

Oil red $O$ staining. RPMs were treated with $50 \mathrm{mg} / \mathrm{l}$ Ox-LDL for 12, 24 and $48 \mathrm{~h}$, washed twice with PBS, and stained with oil red $\mathrm{O}$ and hematoxylin. Intense oil red $\mathrm{O}$ staining (red) was detected in RPMs treated with Ox-LDL, indicating intracellular lipid accumulation. However, staining was markedly reduced in RPMs co-treated with Ox-LDL and $10 \mu \mathrm{M}$ U0126, suggesting reduced lipid deposition (Fig. 1A).

Fluorescence. To examine the effects of the ERK1/2 inhibitor on DiI-Ox-LDL uptake by the RPMs, cells were cultured with DiI-Ox-LDL for 12, 24 and 48 h. Treatment with DiI-Ox-LDL markedly increased cytoplasmic fluorescence of RPMs, as compared with untreated controls (Fig. 1B), and engulfment of DiI-Ox-LDL in RPMs was demonstrated by observation of intracellular accumulation of DiI-labeled lipids. However, markedly reduced DiI-Ox-LDL fluorescence was observed in RPMs following the addition of U0126, indicating that treatment with U0126 may result in reduced lipid deposition.

MTS assay. To evaluate the hypothesis that Ox-LDL may result in necrosis and apoptosis of RPMs, the effect of Ox-LDL on cell viability was evaluated by MTS assay. A greater viability of RPMs was observed following treatment with Ox-LDL at a concentration of $50 \mathrm{mg} / \mathrm{l}$, as compared with Ox-LDL treatment at 10,30, 70 and $90 \mathrm{mg} / \mathrm{l}$ for 12 and $48 \mathrm{~h}$. Treatment with Ox-LDL at a concentration of $50 \mathrm{mg} / \mathrm{l}$ resulted in greater viability of RPMs, as compared with Ox-LDL treatment at 70 and $90 \mathrm{mg} / \mathrm{l}$ for $24 \mathrm{~h}$. These results suggest that cell viability was increased in response to $50 \mathrm{mg} / \mathrm{l} \mathrm{Ox-LDL}$ treatment (Fig. 2A).

Expression levels of ERK and p-ERK. Western blot analysis detected comparative ERK expression in RPMs treated with Ox-LDL for 12, 24 and $48 \mathrm{~h}$, whereas p-ERK expression levels were markedly increased in Ox-LDL-treated RPMs, as compared with untreated RPMs (Fig. 2B). The addition of $10 \mu \mathrm{M} \mathrm{U} 0126$ led to a marked reduction in the expression levels of p-ERK, as compared with treatment with Ox-LDL alone, indicating that the ERK1/2 inhibitor attenuates ERK1/2 phosphorylation.

Inhibition of ERK1/2 upregulates $O x$-LDL-induced ABCA1 expression. To evaluate the effect of ERK1/2 inhibition on ABCA1 expression, mRNA and protein expression levels of ABCA1 were determined in Ox-LDL-treated RPMs. ABCA1 expression levels were markedly elevated in RPMs treated with Ox-LDL alone or Ox-LDL + U0126 at the mRNA (Fig. 3A) and protein (Fig. 3B) level. In addition, the mRNA and protein expression levels of ABCA1 were notably increased in RPMs following co-treatment with Ox-LDL + U0126 for 12 and $24 \mathrm{~h}$; however, the mRNA and protein expression levels of ABCA1 were not significantly greater in RPMs treated with Ox-LDL + U0126 for $48 \mathrm{~h}$, as compared with RPMs treated with Ox-LDL alone. These results indicate that ERK1/2 inhibition may upregulate mRNA and protein expression levels of ABCA1 in Ox-LDL-treated macrophages.

Inhibition of ERK1/2 augments Ox-LDL-induced ABCG1 expression. Western blotting and RT-PCR indicated a significant increase in ABCG1 expression at the transcriptional (Fig. 4A) and translational (Fig. 4B) level following Ox-LDL treatment. In addition, mRNA and protein expression levels of ABCG1 were significantly higher in RPMs co-treated with Ox-LDL and U0126 for 12 and $24 \mathrm{~h}$, as compared with those treated with Ox-LDL alone. Conversely, 

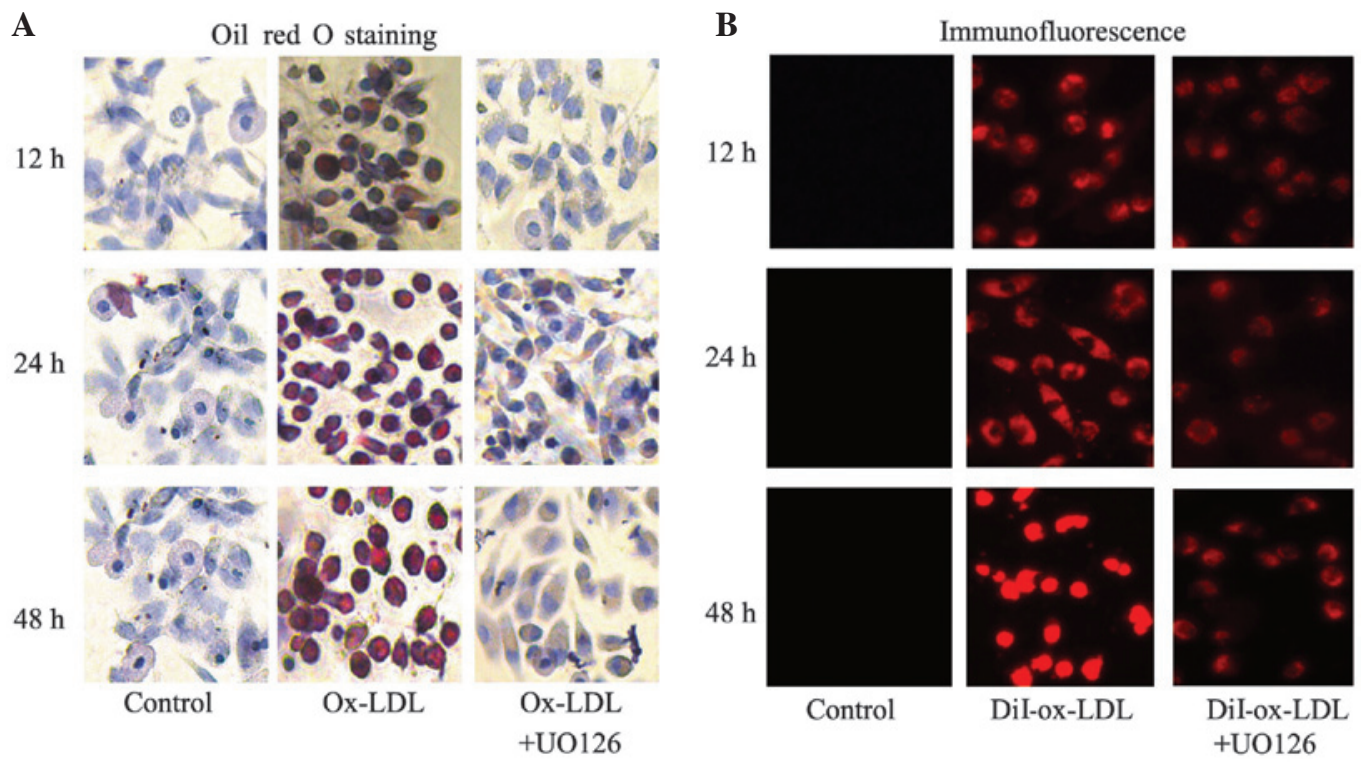

Figure 1. ERK1/2 inhibitor, U0126, markedly reduces lipid deposition in RPMs treated with $50 \mathrm{mg} / \mathrm{l}$ Ox-LDL alone or in combination with $10 \mu \mathrm{M}$ U0126 for 12,24 and 48 h. (A) Cells were stained with oil red $\mathrm{O}$ and examined under an inverted microscope (magnification, $\mathrm{x} 400$ ). RPMs were observed to engulf a large quantity of lipids (red). (B) No red fluorescence was detected in untreated RPMs, whereas red fluorescence was detected in DiI-Ox-LDL-treated RPMs, suggesting lipid deposition in macrophages (magnification, x400). ERK, extracellular signal-regulated kinases; RPMs, rat peritoneal macrophages; Ox-LDL, oxidized-low-density lipoprotein.
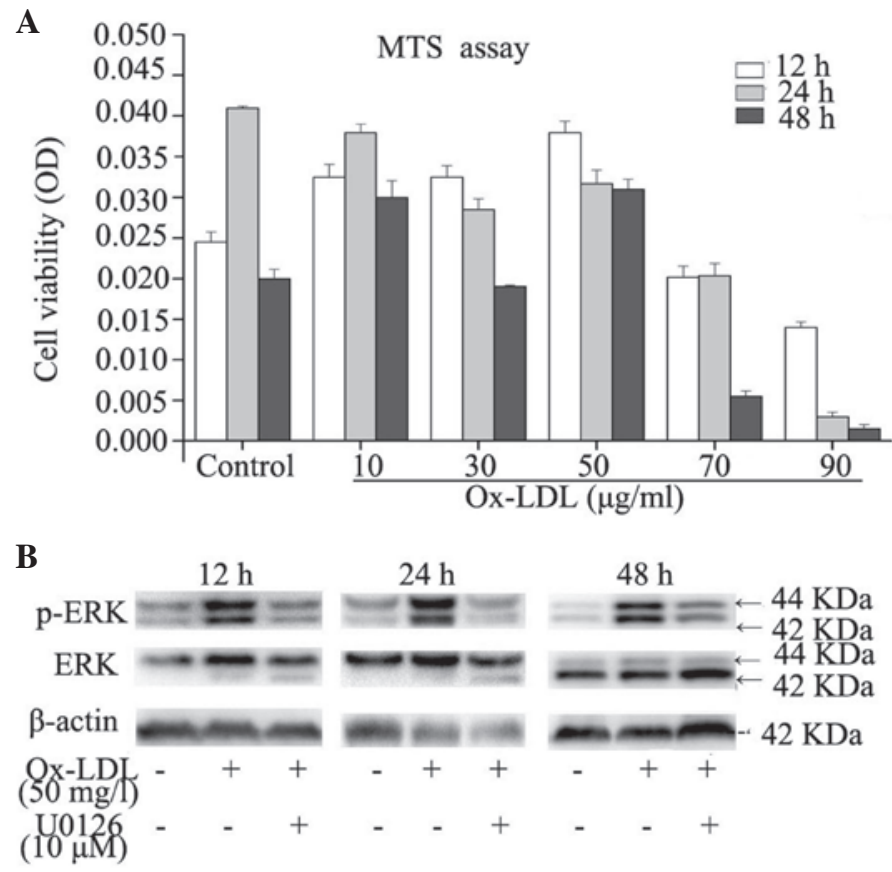

The protein expression of p-ERK, ERK at $12 \mathrm{~h}, 24 \mathrm{~h}, 48 \mathrm{~h}$

Figure 2. Effects of Ox-LDL treatment on ERK and p-ERK protein expression levels in RPMs. (A) RPMs were treated with Ox-LDL at various concentrations for 12, 24 and $48 \mathrm{~h}$. Cell viability was determined by MTS assay. OD demonstrates the viability of cells. (B) RPMs were treated with $50 \mathrm{mg} / \mathrm{l}$ Ox-LDL alone or in combination with $10 \mu \mathrm{M}$ U0126 (ERK inhibitor) for 12, 24 and $48 \mathrm{~h}$, and western blotting was performed to detect ERK and p-ERK protein expression levels. Data are presented as the mean \pm standard deviation. OD, optical density; Ox-LDL, oxidized-low-density lipoprotein; RPMs, rat peritoneal macrophages; ERK, extracellular signal-regulated kinases; p-ERK, phosphorylated ERK.

no significant difference was detected in ABCG1 mRNA and protein expression levels at $48 \mathrm{~h}$ post-treatment with Ox-LDL alone or in combination with U0126, and the mRNA and protein expression levels of ABCG1 were not higher in RPMs treated with Ox-LDL + U0126, as compared with RPMs treated with Ox-LDL alone. These results indicate that
ERK1/2 inhibition augments mRNA and protein expression levels of ABCG1 in Ox-LDL-stimulated macrophages.

Inhibition of ERK1/2 reduces CD36 expression in $O x$ - LDL-treated macrophages. The effect of ERK1/2 inhibition on Ox-LDL-induced CD36 expression was evaluated by 
A

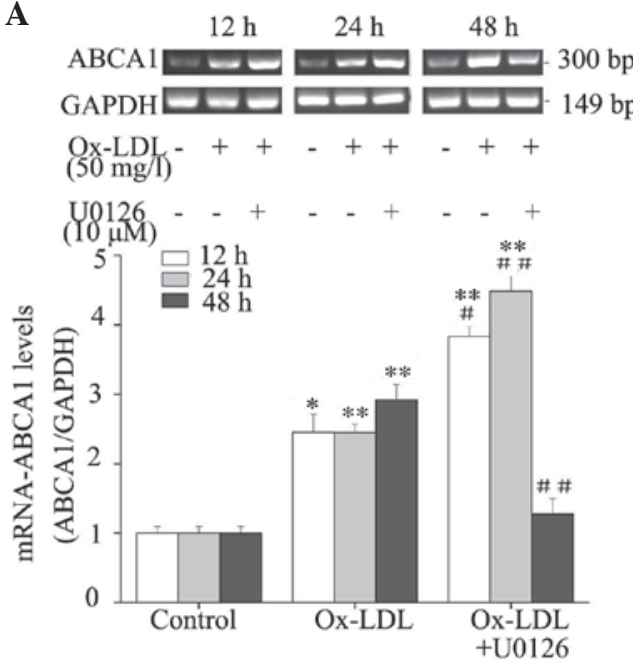

The expression of ABCA1 mRNA at $12 \mathrm{~h}, 24 \mathrm{~h}, 48 \mathrm{~h}$
B
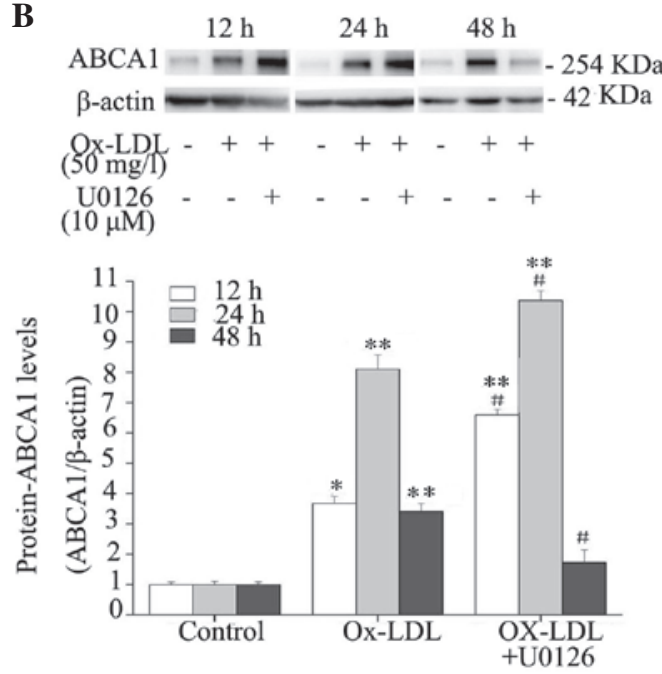

The expression of ABCA1 protein at $12 \mathrm{~h}, 24 \mathrm{~h}, 48 \mathrm{~h}$

Figure 3. ABCA1 mRNA and protein expression levels in RPMs. RPMs were treated with 50 mg/l Ox-LDL alone or in combination with $10 \mu$ M U0126 (ERK inhibitor) for 12, 24 and $48 \mathrm{~h}$. (A) Reverse transcription-polymerase chain reaction was used to determine ABCA1 mRNA expression. GAPDH served as an internal control. (B) Whole cell lysates were used for western blot analysis to determine ABCA1 protein expression. Data are presented as the mean \pm standard deviation. ${ }^{*} \mathrm{P}<0.05,{ }^{* *} \mathrm{P}<0.01$, the Ox-LDL group vs. the control group; ${ }^{\#} \mathrm{P}<0.05,{ }^{\# \#} \mathrm{P}<0.01$, the Ox-LDL + U0126 group vs. the Ox-LDL group. Ox-LDL, oxidized-low-density lipoprotein; ABCA1, ATP-binding cassette transporter A-1; RPMs, rat peritoneal macrophages; GAPDH, glyceraldehyde 3-phosphate dehydrogenase.

A
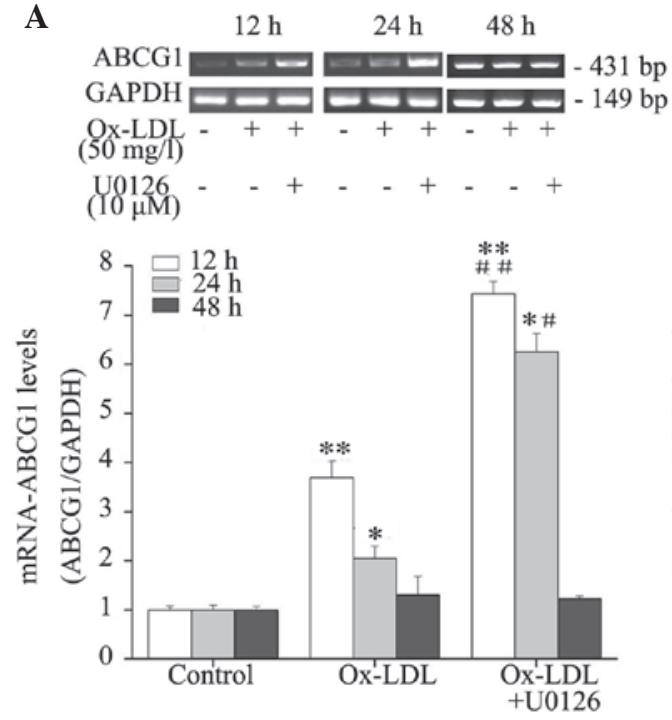

The expression of ABCG1 mRNA at $12 \mathrm{~h}, 24 \mathrm{~h}, 48 \mathrm{~h}$
B
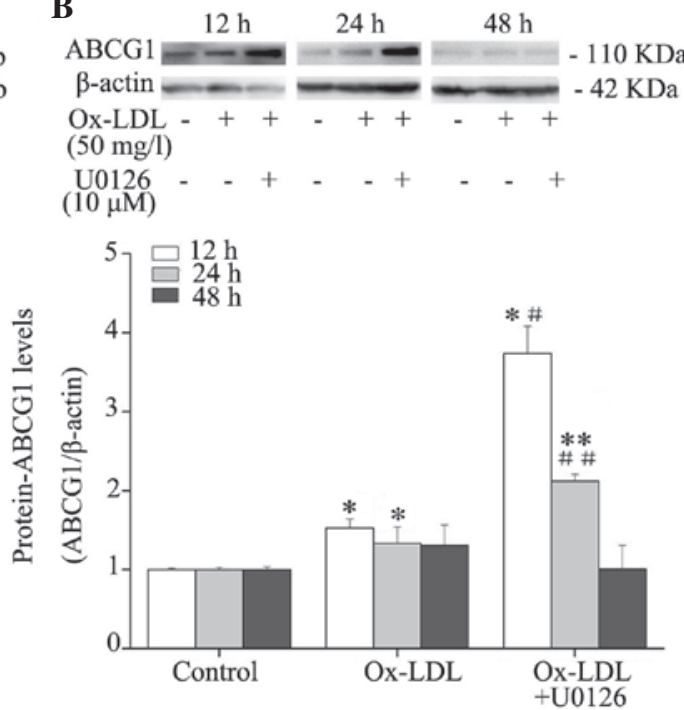

The expression of ABCG1 protein at $12 \mathrm{~h}, 24 \mathrm{~h}, 48 \mathrm{~h}$

Figure 4. Effects of ERK1/2 inhibitor, U0126, on ABCG1 mRNA and protein expression levels in RPMs. RPMs were treated with 50 mg/1 Ox-LDL alone or in combination with $10 \mu \mathrm{M}$ U0126 for 12, 24 and 48 h. (A) Reverse transcription-polymerase chain reaction was used to determine ABCG1 mRNA expression. GAPDH served as an internal control. (B) Whole cell lysates were used for western blot analysis to determine protein expression. Data are presented as the mean \pm standard deviation. ${ }^{*} \mathrm{P}<0.05,{ }^{* *} \mathrm{P}<0.01$, the Ox-LDL group vs. the control group; ${ }^{\#} \mathrm{P}<0.05$, ${ }^{\# \#} \mathrm{P}<0.01$, the Ox-LDL $+\mathrm{U} 0126$ group vs. the Ox-LDL group. RPMs, rat peritoneal macrophages; ERK, extracellular signal-regulated kinases; Ox-LDL, oxidized-low-density lipoprotein; ABCG1, ATP-binding cassette transporter G-1; GAPDH, glyceraldehyde 3-phosphate dehydrogenase.

RT-PCR and western blotting. Treatment with Ox-LDL resulted in a significant elevation in the expression levels of CD36, as compared with the untreated controls $(\mathrm{P}<0.05)$, and co-treatment with Ox-LDL and U0126 for 12, 24 and $48 \mathrm{~h}$ resulted in significant reductions in mRNA (Fig. 5A) and protein (Fig. 5B) expression levels of CD36, as compared with treatment with Ox-LDL alone. These results suggest that Ox-LDL markedly induces CD36 expression and inhibition of ERK1/2 reduces CD36 expression in Ox-LDL-treated macrophages.

\section{Discussion}

Atherosclerotic lesions are characterized by accumulation of cholesterol in the arterial tunica intima. Macrophages are central to the development and progression of AS $(13,14)$; they have been observed to remove cholesterol deposits in arteries, which is beneficial in the initial stages of AS; however, continued cholesterol accumulation may result in the formation of foam cells, which are involved in AS progression $(15,16)$. 
A
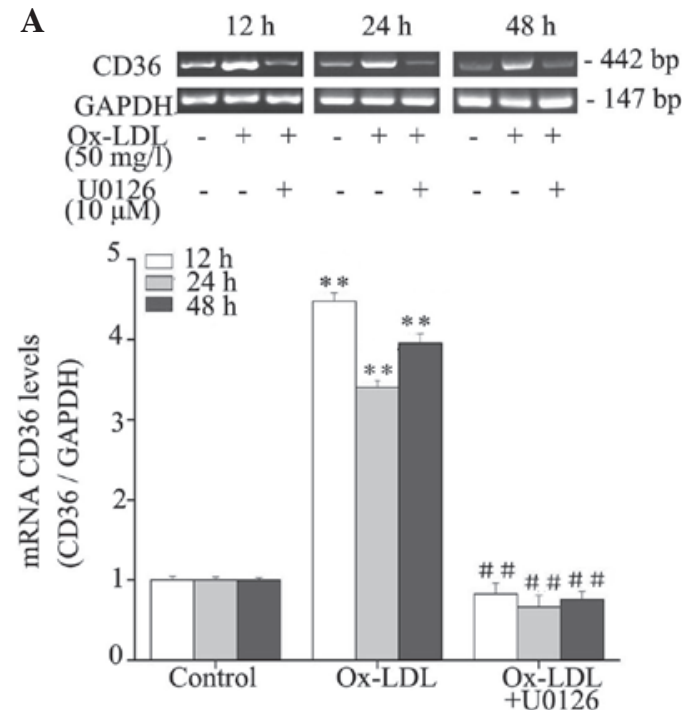

The expression of CD36 mRNA at $12 \mathrm{~h}, 24 \mathrm{~h}, 48 \mathrm{~h}$

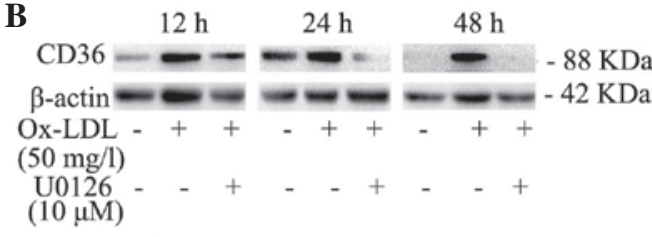

$(10 \mu \mathrm{M}$

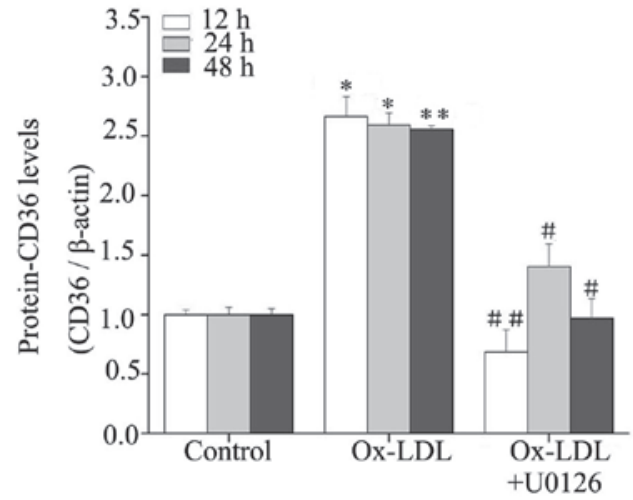

The expression of CD36 protein at $12 \mathrm{~h}, 24 \mathrm{~h}, 48 \mathrm{~h}$

Figure 5. Effects of ERK1/2 inhibitor, U0126, on CD36 mRNA and protein expression levels in RPMs. RPMs were treated with 50 mg/l Ox-LDL alone or in combination with $10 \mu \mathrm{M}$ U0126 for 12, 24 and $48 \mathrm{~h}$. (A) Reverse transcription-polymerase chain reaction was used to determine CD36 mRNA expression. GAPDH served as an internal control. (B) Whole cell lysates were used for western blot analysis to determine CD36 protein expression. Data are presented as the mean \pm standard deviation. ${ }^{*} \mathrm{P}<0.05,{ }^{* *} \mathrm{P}<0.01$, the Ox-LDL group vs. the control group; ${ }^{\#} \mathrm{P}<0.05,{ }^{\# \#} \mathrm{P}<0.01$, the Ox-LDL $+\mathrm{U} 0126$ group vs. the Ox-LDL group. CD, cluster of differentiation; Ox-LDL, oxidized-low-density lipoprotein; RPMs, rat peritoneal macrophages; ERK, extracellular signal-regulated kinases; GAPDH, glyceraldehyde 3-phosphate dehydrogenase.

The response of RPMs to modified LDL is considered a good model for AS research $(17,18)$. The results of the present study indicated that RPMs engulfed a large quantity of Ox-LDL, as demonstrated by DiI fluorescence and oil red $\mathrm{O}$ staining. In addition, the present study demonstrated that inhibition of ERK1/2 markedly reduced Ox-LDL deposition in the RPMs. It has previously been reported that ERK1/2 is associated with cell migration, survival, differentiation and proliferation (4). However, the association between ERK1/2 and lipid metabolism remains to be elucidated.

Cholesterol uptake is a pathway by which extracellularly modified LDLs are ingested by macrophages via scavenger receptors. As a major scavenger receptor of Ox-LDL $(19,20)$, CD36 binds to $>50 \%$ of Ox-LDL in macrophages (21), which is crucial to the progression of AS. Absence of CD36 on the cell surface of macrophages has been demonstrated to be protective against AS (22). In addition, treatment with a CD36 competitive peptide ligand (EP80317) that blocks the Ox-LDL-binding site of CD36 resulted in a marked reduction (up to 51\%) of atherosclerotic lesions in apolipoprotein E-deficient mice (23). It has therefore been hypothesized that inhibition of CD36 expression in macrophages has potential anti-atherosclerotic effects. It has been reported that the CD36 signaling pathway may be initiated by internalization of Ox-LDL $(3,7)$. Consistent with these previous findings, the present study indicated that Ox-LDL resulted in a notable increase in CD36 expression. Increasing evidence suggests that Ox-LDL increases ERK phosphorylation $(3,20,24)$. The results of the present study indicated that inhibition of ERK1/2 markedly reduced Ox-LDL-induced CD36 expression in RPMs. Furthermore, a p38 MAPK inhibitor, SB203580, has previously been demonstrated to suppress CD36 expression in THP-1 cell lines (24) and RAW264.7 cells (20). The present study demonstrated that treatment with an ERK1/2 inhibitor, U0126, attenuated Ox-LDL-induced CD36 expression at the transcriptional and translational level. It is therefore assumed that ERK1/2 may be important in mediating CD36 expression in macrophages.

Macrophages have been reported to maintain cellular lipid homeostasis via cholesterol efflux pathways. The principle molecules associated with cholesterol efflux in macrophages are ABCA1 and ABCG1 $(25,26)$. In THP-1 cells, Ox-LDL has been shown to upregulate ABCA1 expression (27). The present study demonstrated that the mRNA and protein expression levels of ABCA1 were significantly increased following Ox-LDL treatment, and that inhibition of ERK1/2 increased ABCA1 mRNA and protein expression levels in macrophages. It has been reported that ERK1/2 inhibitors synergize with LXR activation, in order to induce ABCA1 expression in a RAW macrophage cell line (5), and U0126, an ERK1/2 inhibitor, has been demonstrated to delay the degradation of ABCA1 mRNA and protein in macrophages (28). However, a previous study indicated that inhibition of ERK1/2 was able to promote ABCA1 and ABCG1 protein degradation in tumor cells, whereas inhibition of ERK1/2 upregulated ABCA1 and ABCG1 expression in human THP-1 macrophages (29). It is therefore hypothesized that the effect of ERK1/2 inhibition on ABCA1 and ABCG1 expression may depend on cell specificity. The results of the present study demonstrated that inhibition of ERK1/2 increased ABCA1 expression in Ox-LDL-induced macrophages at 12 and $24 \mathrm{~h}$, and gradually decreased ABCA1 expression at $48 \mathrm{~h}$. The half-life of ABCA1 protein is 1-2 $\mathrm{h}$, and ABCA1 is frequently present during homeostasis between expression and degradation. Inhibition of ERK1/2 has been demonstrated to suppress the degradation of ABCA1 mRNA and protein (5) or increase its stability (5). However, macro- 
phages secrete various inflammatory cytokines, which affect ABCA1 expression (30,31). Further studies are required to investigate the mechanisms underlying the effects of ERK1/2 inhibition on the regulation of cytokines that affect ABCA1 expression. The present study demonstrated that alterations to ABCG1 mRNA and protein expression levels were similar to those observed in ABCA1 expression at 12 and $24 \mathrm{~h}$ post-treatment with Ox-LDL. It has been reported that macrophages may overexpress unsaturated fatty acids and products of 12/15-lipoxygenase, which increase the degradation of ABCG1 protein $(32,33)$. In addition, ABCG1 expression is affected by cell activity and cytokines secreted from macrophages (34).

The present study indicated that inhibition of ERK1/2 markedly suppressed lipid deposition in macrophages by promoting ABCA1 and ABCG1 expression, which are associated with cholesterol efflux. ABCA1 and ABCG1 expression may be induced by ERK1/2 inhibition or $\operatorname{LXR} \alpha$ (35), thus indicating that an association may exist between LXR and ERK. Since ABCA1 and ABCG1 are nuclear receptor LXR target genes involved in cholesterol efflux, activation of LXR may increase ABCA1 and ABCG1 expression in macrophages $(36,37)$. However, it has been reported that ERK1/2 inhibitors have no effect on LXR $\alpha$ and LXR $\beta$ expression (5). It is conceivable that ERK1/2 inhibition may directly increase ABCA1 and ABCG1 expression by methods other than upregulating LXR expression. ERK1/2 controls transcriptional and post-transcriptional regulation of LXR and peroxisome proliferator-activated receptor (PPAR) $\alpha / \gamma(29,38-40)$, and mediates expression of their target genes. Further studies are required to elucidate the association between ERK and PPARs/LXRs-ABCA1/G1, and to understand the underlying mechanisms.

In conclusion, inhibition of ERK1/2 increases macrophage lipid efflux by upregulating ABCA1 and ABCG1 expression, and suppresses lipid engulfment by downregulating CD36 expression at the transcriptional and translational level. These findings suggest that inhibition of ERK1/2 may exert potential anti-atherosclerotic effects, and involvement of the ERK1/2 pathway in lipid metabolism may provide additional knowledge for the development of novel treatment strategies for AS.

\section{Acknowledgements}

The present study was supported by grants from the National Natural Science Foundation of China (grant nos. 81001543 and 81473744) and the Personal Supporting Projects of Health Department in Fujian Province of China (grant no. 2013-ZQN-ZD-28). The funders had no role in study design, data collection and analysis, decision to publish or preparation of the manuscript.

\section{References}

1. Lusis AJ: Atherosclerosis. Nature 407: 233-241, 2000.

2. Febbraio M and Silverstein RL: CD36: Implications in cardiovascular disease. Int J Biochem Cell Biol 39: 2012-2030, 2007.

3. Rahaman SO, Lennon DJ, Febbraio M, Podrez EA, Hazen SL and Silverstein RL: A CD36-dependent signaling cascade is necessary for macrophage foam cell formation. Cell Metab 4: 211-221, 2006

4. Roskoski RJ Jr: ERK1/2 MAP kinases: Structure, function and regulation. Pharmacol Res 66: 105-143, 2012.
5. Zhou X, Yin Z, Guo X, Hajjar DP and Han J: Inhibition of ERK1/2 and activation of liver $X$ receptor synergistically induce macrophage ABCA1 expression and cholesterol efflux. J Biol Chem 285: 6316-6326, 2010.

6. Ye D, Lammers B, Zhao Y, Meurs I, Van Berkel TJ and Van Eck M: ATP-binding cassette transporters A1 and G1, HDL metabolism, cholesterol efflux, and inflammation: Important targets for the treatment of atherosclerosis. Curr Drug Targets 12: 647-660, 2011.

7. Silverstein RL and Febbraio M: CD36 and atherosclerosis. Curr Opin Lipidol 11: 483-491, 2000.

8. Liu HY, Cui HB, Chen XM, Chen XY, Wang SH, Du WP, Zhou HL, Zhao RC, Zhou Y, Liu YH, et al: Imbalanced response of ATP-binding cassette transporter A1 and CD36 expression to increased oxidized low-density lipoprotein loading contributes to the development of THP-1 derived foam cells. J Biochem 155: 35-42, 2014

9. Liu W, Jiang J, Yan D, Li D, Li W, Ma Y, Yang L, Qu Z and Ruan Q: Pentraxin 3 promotes oxLDL uptake and inhibits cholesterol efflux from macrophage-derived foam cells. Exp Mol Pathol 96: 292-299, 2014.

10. Jing Q, Xin SM, Zhang WB, Wang P, Qin YW and Pei G: Lysophosphatidylcholine activates p38 and p42/44 mitogen-activated protein kinases in monocytic THP-1 cells, but only p38 activation is involved in its stimulated chemotaxis. Circ Res 87: 52-59, 2000.

11. Cheng X, Liu X, Song L, He Y, Li X and Zhang H: Atorvastatin inhibits macrophage-derived foam cell formation by suppressing the activation of PPAR $\gamma$ and NF- $\kappa$ B pathway. Nan Fang Yi Ke Da Xue Xue Bao 34: 896-900, 2014 (In Chinese).

12. Bhandary B, Lee GH, So BO, Kim SY, Kim MG, Kwon JW, Song JY, Lee HK, Kim HR, Chae SW and Chae HJ: Rubus coreanus inhibits oxidized-LDL uptake by macrophages through regulation of JNK activation. Am J Chin Med 40: 967-978, 2012.

13. Glass CK and Witztum JL: Atherosclerosis. The road ahead. Cell 104: 503-516, 2001.

14. Tiwari RL, Singh V and Barthwal MK: Macrophages: An elusive yet emerging therapeutic target of atherosclerosis. Med Res Rev 28: 483-544, 2008.

15. Ouimet $\mathrm{M}$ : Autophagy in obesity and atherosclerosis: Interrelationships between cholesterol homeostasis, lipoprotein metabolism and autophagy in macrophages and other systems. Biochim Biophys Acta 1831: 1124-1133, 2013.

16. Webb NR and Moore KJ: Macrophage-derived foam cells in atherosclerosis: Lessons from murine models and implications for therapy. Curr Drug Targets 8: 1249-1263, 2007.

17. Morio H, Saito H, Hirai A, Tamura Y and Yoshida S: Effect of modified LDL on the release of NO and PGI2 from rat peritoneal macrophages. J Atheroscler Thromb 2: 41-45, 1995.

18. Hirai A, Kino T, Tokinaga K, Tahara K, Tamura Y and Yoshida S: Regulation of sterol carrier protein 2 (SCP2) gene expression in rat peritoneal macrophages during foam cell formation. A key role for free cholesterol content. J Clin Invest 94: 2215-2223, 1994.

19. Nicholson AC and Hajjar DP: CD36, oxidized LDL and PPAR gamma: Pathological interactions in macrophages and atherosclerosis. Vascul Pharmacol 41: 139-146, 2004.

20. Min KJ, Um HJ, Cho KH and Kwon TK: Curcumin inhibits oxLDL-induced CD36 expression and foam cell formation through the inhibition of p38 MAPK phosphorylation. Food Chem Toxicol 58: 77-85, 2013.

21. Febbraio M, Podrez EA, Smith JD, Hajjar DP, Hazen SL, Hoff HF, Sharma K and Silverstein RL: Targeted disruption of the class B scavenger receptor CD36 protects against atherosclerotic lesion development in mice. J Clin Invest 105: 1049-1056, 2000.

22. Febbraio M, Guy E and Silverstein RL: Stem cell transplantation reveals that absence of macrophage CD36 is protective against atherosclerosis. Arterioscler Thromb Vasc Biol 24: 2333-2338, 2004.

23. Marleau S, Harb D, Bujold K, Avallone R, Iken K, Wang Y, Demers A, Sirois MG, Febbraio M, Silverstein RL, et al: EP 80317, a ligand of the CD36 scavenger receptor, protects apolipoprotein E-deficient mice from developing atherosclerotic lesions. FASEB J 19: 1869-1871, 2005.

24. Zhao M, Liu Y, Wang X, New L, Han J and Brunk UT: Activation of the p38 MAP kinase pathway is required for foam cell formation from macrophages exposed to oxidized LDL. APMIS 110: 458-468, 2002. 
25. Wang N, Lan D, Chen W, Matsuura F and Tall AR: ATP-binding cassette transporters G1 and G4 mediate cellular cholesterol efflux to high-density lipoproteins. Proc Natl Acad Sci USA 101: 9774-9779, 2004

26. Wang N, Silver DL, Costet $P$ and Tall AR: Specific binding of ApoA-I, enhanced cholesterol efflux, and altered plasma membrane morphology in cells expressing ABC1. J Biol Chem 275: 33053-33058, 2000.

27. Tang CK, Yi GH, Yang JH, Liu LS, Wang Z, Ruan CG and Yang YZ: Oxidized LDL upregulated ATP binding cassette transporter-1 in THP-1 macrophages. Acta Pharmacol Sin 25: 581-586, 2004.

28. Chang YC, Sheu WH, Chien YS, Tseng PC, Lee WJ and Chiang AN: Hyperglycemia accelerates ATP-binding cassette transporter A1 degradation via an ERK-dependent pathway in macrophages. J Cell Biochem 114: 1364-1373, 2013.

29. Mulay V, Wood P, Manetsch M, Darabi M, Cairns R, Hoque M, Chan KC, Reverter M, Alvarez-Guaita A, Rye KA, et al: Inhibition of mitogen-activated protein kinase Erk1/2 promotes protein degradation of ATP binding cassette transporters A1 and $\mathrm{G} 1$ in $\mathrm{CHO}$ and HuH7 cells. PLoS One 8: e62667, 2013

30. Hao XR, Cao DL, Hu YW, Li XX, Liu XH, Xiao J, Liao DF, Xiang J and Tang CK: IFN-gamma down-regulates ABCA1 expression by inhibiting LXRalpha in a JAK/STAT signaling pathway-dependent manner. Atherosclerosis 203: 417-428, 2009.

31. Chen M, Li W, Wang N, Zhu Y and Wang X: ROS and NF-kappaB but not LXR mediate IL-1beta signaling for the downregulation of ATP-binding cassette transporter A1. Am J Physiol Cell Physiol 292: C1493-C1501, 2007.

32. Uehara Y, Miura S, von Eckardstein A, Abe S, Fujii A, Matsuo Y, Rust S, Lorkowski S, Assmann G, Yamada T and Saku K: Unsaturated fatty acids suppress the expression of the ATP-binding cassette transporter G1 (ABCG1) and ABCA1 genes via an LXR/RXR responsive element. Atherosclerosis 191: $11-21,2007$.
33. Nagelin MH, Srinivasan S, Lee J, Nadler JL and Hedrick CC: 12/15-Lipoxygenase activity increases the degradation of macrophage ATP-binding cassette transporter G1. Arterioscler Thromb Vasc Biol 28: 1811-1819, 2008.

34. Yang C, Cui K, Diao Y, Du M and Wang S: Effect of selenium-enriched garlic pil against cytotoxicity induced by OX-LDL in endothelial cells. Evid Based Complement Alternat Med 2014: 537652, 2014.

35. Xue X, Chen T, Wei W, Zhou X, Lin Z and Chen L: Effects of Alisma Decoction on lipid metabolism and inflammatory response are mediated through the activation of the LXRo pathway in macrophage-derived foam cells. Int J Mol Med 33: 971-977, 2014

36. Tang SL, Chen WJ, Yin K, Zhao GJ, Mo ZC, Lv YC, Ouyang XP, Yu XH, Kuang HJ, Jiang ZS, et al: PAPP-A negatively regulates ABCA1, ABCG1 and SR-B1 expression by inhibiting LXR $\alpha$ through the IGF-I-mediated signaling pathway. Atherosclerosis 222: 344-354, 2012

37. Ma AZ, Song ZY and Zhang Q: Cholesterol efflux is LXR $\alpha$ isoform-dependent in human macrophages. BMC Cardiovasc Disord 14: 80, 2014.

38. Bhatt KH, Sodhi A and Chakraborty R: Peptidoglycan induced expression of peroxisome proliferator-activated receptor $\gamma$ in mouse peritoneal macrophages: Role of ERK and JNK MAP kinases. Cytokine 60: 778-786, 2012.

39. Stachowska E, Kijowski J, Dziedziejko V, Siennicka A and Chlubek D: Conjugated linoleic acid regulates phosphorylation of PPAR $\gamma$ by modulation of ERK $1 / 2$ and p38 signaling in human macrophages/fatty acid-laden macrophages. J Agric Food Chem 59: 11846-11852, 2011.

40. Mogilenko DA, Shavva VS, Dizhe EB, Orlov SV and Perevozchikov AP: PPAR $\gamma$ activates ABCA1 gene transcription but reduces the level of ABCA1 protein in HepG2 cells. Biochem Biophys Res Commun 402: 477-482, 2010. 